\title{
Wavefront aberration analysis with a multi-order diffractive optical element
}

\author{
P.A. Khorin ${ }^{1}$, S.A. Degtyarev ${ }^{1,2}$ \\ ${ }^{1}$ Samara National Research University, 34 Moskovskoe Shosse, 443086, Samara, Russia \\ ${ }^{2}$ Image Processing Systems Institute - Branch of the Federal Scientific Research Centre "Crystallography and Photonics" of Russian Academy of Sciences, 151 \\ Molodogvardeyskaya st., 443001, Samara, Russia
}

\begin{abstract}
In this paper we show an ability to use a multi-order (multi-channel) diffractive optical element for wavefront relief function expansion in terms of Zernike polynomials. This approach can be successfully used for small meanings of wavefront aberrations when the wavefront relief can be represented as a linear superposition of Zernike polynomials. Unfortunately, linear approximation is becoming unworkable with increasing of aberration meanings. In this work we study an applicability of this Zernike expansion method.
\end{abstract}

Keywords: Zernike polynomials; measurements of wavefront aberrations; multi-order diffractive optical element

\section{Introduction}

Light field phase retrieval is one of the fundamental problems of signal analysis processing [1]. Nowadays we do not have a direct method of phase registration; consequently phase can be measured indirectly through light intensity analysis. For example, wavefront can be retrieved with interferometrical methods accompanied by subsequent calculation algorithms. Once more method is implemented in Shack-Hartmann wavefront sensor. Usually, it consists of an array of pinholes or microlenses; each of them plots a tilt of analyzed wavefront into sensor matrix [2]. Phase retrieval can be also implemented with diffractive optical elements (DOEs) which can expand analyzed light field into an orthogonal basis [3-5].

Commonly used wavefront representation is an expansion into Zernike polynomials [6]. Weight coefficients of the wavefront expansion make it possible to calculate root-mean-square deviation from the ideal spherical wavefront. In this case every weight coefficient is associated with a certain aberration. Thus, the weight coefficient with high value automatically points into an aberration which mostly deforms the wavefront.

In this work we propose a diffractive optical element (DOE) which is matched with Zernike polynomials basis [7, 8, 9]. This element has been successfully used for weakly aberrated wavefront analysis [10-11].

Each Zernike polynomial corresponds to a certain optical wavefront aberration. Conventional wavefront aberration type can be described as a certain Zernike polynomial with orders $n$ and $m$ like it is shown in Table 1.

Table 1. Correspondence between Zernike polynomials and conventional wavefront aberration types.

\begin{tabular}{ccccc}
\hline No & $n$ & $m$ & Zernike polynomial & Aberration type \\
\hline $\mathbf{1}$ & 0 & 0 & 1 & Constant \\
\hline $\mathbf{2}$ & 1 & -1 & $2 r \sin (\theta)$ & Tilt \\
\hline $\mathbf{3}$ & 1 & 1 & $2 r \cos (\theta)$ & Tilt \\
\hline $\mathbf{4}$ & 2 & -2 & $\sqrt{6} r^{2} \sin (2 \theta)$ & Astigmatism \\
\hline $\mathbf{5}$ & 2 & 0 & $\sqrt{3}\left(2 r^{2}-1\right)$ & Defocus \\
\hline $\mathbf{6}$ & 2 & 2 & $\sqrt{6} r^{2} \cos (2 \theta)$ & Astigmatism \\
\hline $\mathbf{7}$ & 3 & -3 & $2 \sqrt{2} r^{3} \sin (3 \theta)$ & (Trefoil) \\
\hline $\mathbf{8}$ & 3 & -1 & $2 \sqrt{2}\left(3 r^{3}-2 r\right) \sin (\theta)$ & Pure coma \\
\hline $\mathbf{9}$ & 3 & 1 & $2 \sqrt{2}\left(3 r^{3}-2 r\right) \cos (\theta)$ & Pure coma \\
\hline $\mathbf{1 0}$ & 3 & 3 & $2 \sqrt{2} r^{3} \cos (3 \theta)$ & (Trefoil) \\
\hline $\mathbf{1 1}$ & 4 & -4 & $\sqrt{10} r^{4} \sin (4 \theta)$ & Quadrofoll \\
\hline $\mathbf{1 2}$ & 4 & -2 & $\sqrt{10}\left(4 r^{4}-3 r^{2}\right) \sin (2 \theta)$ & $2^{\text {th }}$ order Astigmatism \\
\hline $\mathbf{1 3}$ & 4 & 0 & $\sqrt{5}\left(6 r^{4}-6 r^{2}+1\right)$ & Spherical \\
\hline $\mathbf{1 4}$ & 4 & 2 & $\sqrt{10}\left(4 r^{4}-3 r^{2}\right) \cos (2 \theta)$ & $2^{\text {th }}$ order Astigmatism \\
\hline $\mathbf{1 5}$ & 4 & 4 & $\sqrt{10} r^{4} \cos (4 \theta)$ & Quadrofoll \\
\hline
\end{tabular}

In this work we assume that Zernike polynomials appear as follows:

$$
\psi_{n m}(r, \varphi)=\sqrt{\frac{n+1}{\pi r_{0}^{2}}} R_{n}^{m}(r)\left\{\begin{array}{c}
\cos (m \varphi) \\
\sin (m \varphi)
\end{array}\right\},
$$


here $R_{n}^{m}(r)$ is radial Zernike polynomials:

$$
R_{n}^{m}(r)=\sum_{p=0}^{(n-m) / 2} \frac{(-1)^{p}(n-p) !}{p !\left(\frac{n+m}{2}-p\right) !\left(\frac{n-m}{2}-p\right) !}\left(\frac{r}{R}\right)^{n-2 p}
$$

Wavefront aberrations $W(r, \varphi)$ are commonly described in terms of Zernike polynomials in the following way:

$$
\begin{aligned}
& W(r, \varphi)=\exp [i w(r, \varphi)], \\
& w(r, \varphi)=\sum_{n=0}^{N} \sum_{m=-n}^{n} c_{n m} \psi_{n m}(r, \varphi) .
\end{aligned}
$$

There is so-called "Zernike pyramid" in figure 1. 2D patterns of few Zernike polynomials form this pyramid. In a vertical direction radial number $n$ varies from 0 to $4(n=0$ to 4$)$ and in a horizontal direction azimuthal number $m$ varies from $-n$ to $n$ $(m=-n$ to $n)$.

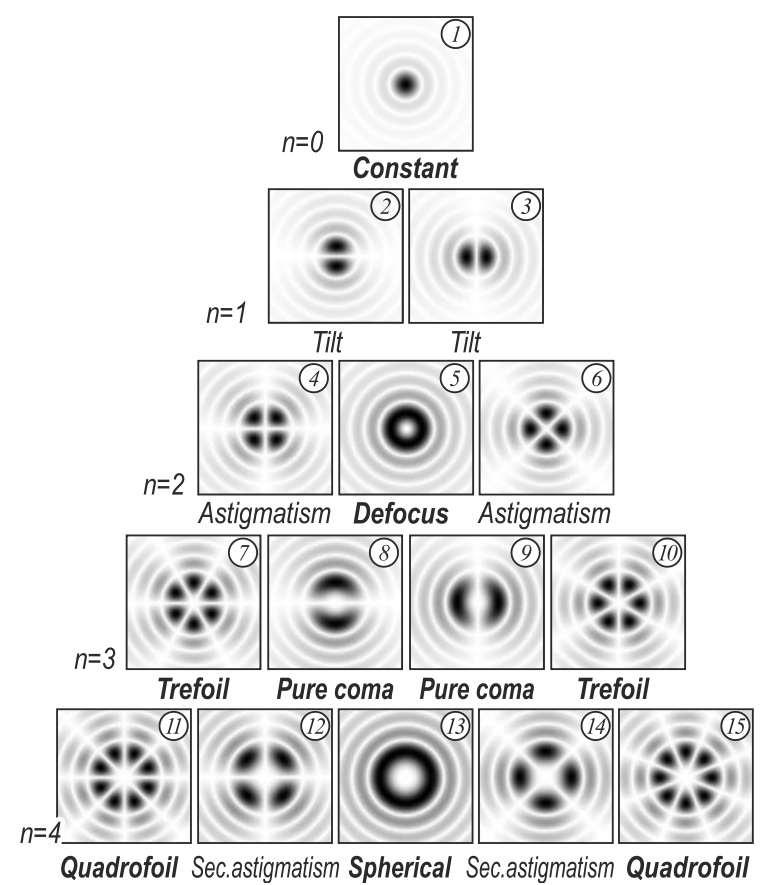

Fig. 1. Zernike pyramid: 2D pattern of few first Zernike polynomials.

For plotting the point-spread function we use a model of Fourier optical correlator (figure 2). This simple optical arrangement is simulated with Zemax ray-tracing software [12].

Control of optical image quality involves measurements optical system image photometric parameters such as spread function of optical system (for example, point-spread function). Practically, these image photometric parameters quantitatively characterize optical system image quality. State-of-the-art theory has been carefully developed, thus, a certain set of parameters completely describes the quality of an optical system image.

Experimentally received point-spread function characterizes the quality of an optical system. It describes quite thoroughly optical system characteristics including wavefront and optical surfaces microrelief.

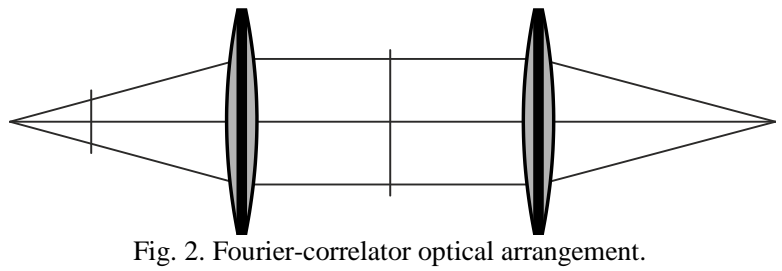

Optical elements work together and create an image. Unfortunately, created with an optical system image can not be ideal. Lenses and mirrors have their own aberrations; in addition, aberrations can arise due to inaccuracies of fabrication, misalignments, and so on. Partial error compensation can be provided after precise measurements of wavefront aberrations.

In our simulations we introduce aberrations into the optical system through the adding to the relief of the first surface of the second lens. These approach and algorithm are discussed in detail in paper [13]. 2D patterns of point-spread function are shown in figure 3. This patterns are arranged as a pyramid corresponding to Zernike pyramid in figure 1. 


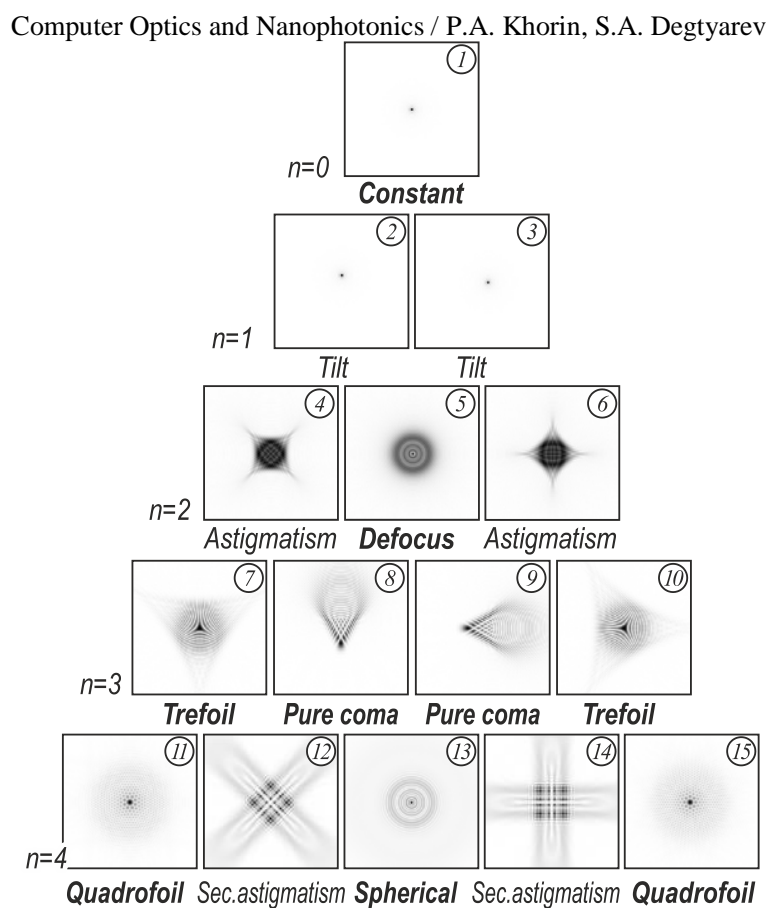

Fig. 3. Point-spread functions 2D patterns that are corresponding to conventional aberration types.

\section{Wavefront aberration analysis}

In the paper [14] authors investigate an ability of using multi-order diffractive optical element for analysis of human eye optical system aberrations $[15,16]$. Note that, this way may be used for other optical systems, including telescopes [17-19].

It should be noticed that Zernike bases can differ not only in indexing and normalization but in the angular dependence shape. In particular, in [20] exponential and trigonometric angular dependences are used for designing multi-order diffractive optical elements for wavefront analysis.

Optical Zernike analyzer is a combination of diffractive optical element and lens; DOE is matched with basis function and lens does Fourier transform. Achieved Fourier spectrum at the center has the value which means scalar product of input light function and the basis function. Experimental testing of the same devise is described in [20, 21].

Certain basis Zernike function is coded in each order of multi-order diffractive optical element. DOE transmission function looks as follows:

$$
\tau(x, y)=\sum_{p=0}^{P} \sum_{q=0}^{Q} \psi_{p q}^{*}(x, y) \exp \left[i\left(\alpha_{p q} x+\beta_{p q} y\right)\right]
$$

Amplitude and phase 2D patterns of 8-order DOE transmission function are shown in fig. 4.
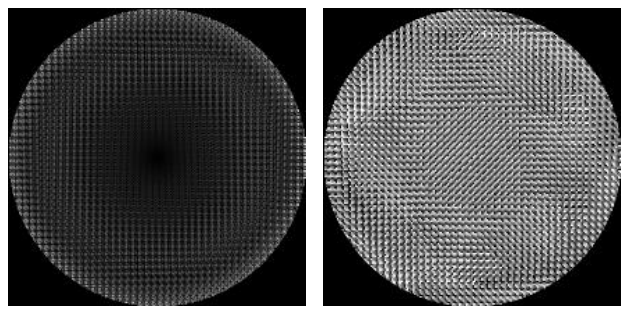

Fig. 4. Amplitude and phase 2D patterns of 8-order DOE transmission function.

There is an illustration of 8-order diffractive optical filter working in figure 5. Plane wave (wavefront is flat $w(x, y)=1$ ) illuminates the element and diffracts on it. As can be seen, all orders are holed. It means that analyzed wavefront does not have any aberrations.

In this work we propose a physical model of optical device (figure 6) for wavefront aberration analysis. This device is based on multi-order Zernike-matched diffractive optical element. Analyzed wavefront $w(x, y)$ passes through the element. The lens $O$ with focal distance $f$ makes Fourier transform. Sensor matrix should be posed at the focal distance from the lens. Sensor plane has $u$ and $v$ coordinate axes. 
Computer Optics and Nanophotonics / P.A. Khorin, S.A. Degtyarev

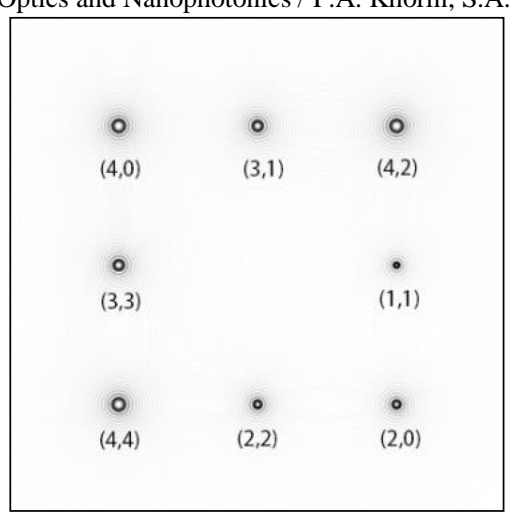

Fig.5. Plane wave filtering with 8-order diffractive optical filter.

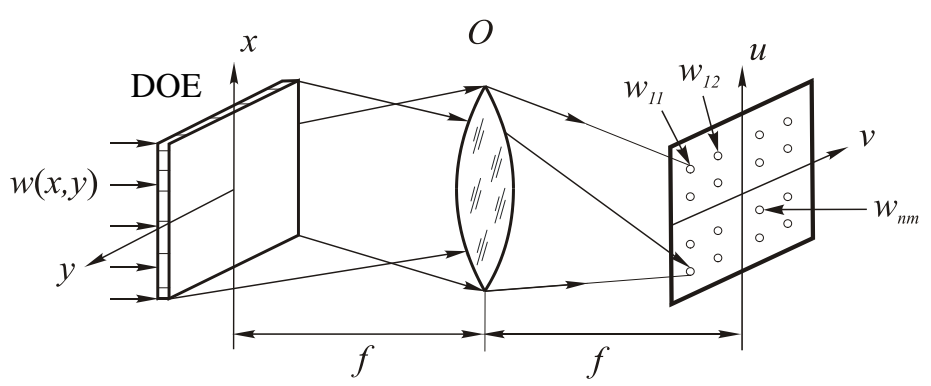

Fig.6. Optical scheme of proposed aberration analyzer.

Another testing simulation of proposed Zernike analyzer model is provided for initial beam wavefront $w(x, y)=\psi_{2,0}(x, y)+$ $\psi_{2,2}(x, y)$. Resulting pattern is shown in fig. 7. Simulation shows that 8 -order filter detects defocusing and astigmatism aberrations as it is set for initial beam. Detected coefficient meanings are $C_{20}=0.995, C_{22}=0.996$ but in initial beam they are equal to 1 .

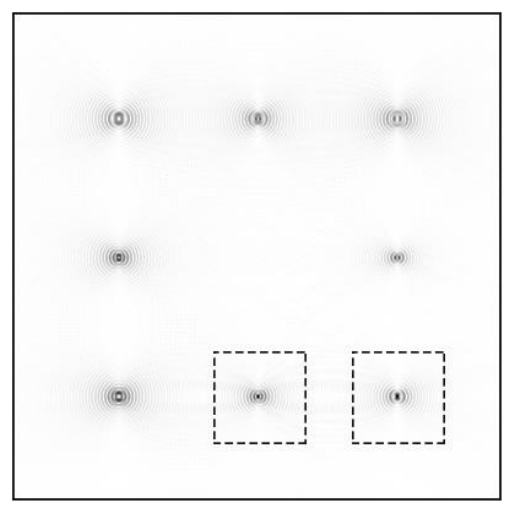

Fig. 7. Resulting amplitude patterns which is formed with 8 -order analyzer after filtering the initial wavefront $w(x, y)=\psi_{2,0}(x, y)+\psi_{2,2}(x, y)$.

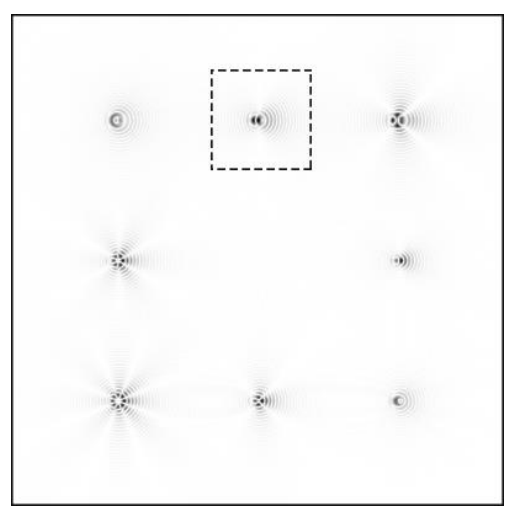

Fig. 8. Resulting amplitude patterns which is formed with 8-order analyzer after filtering the initial wavefront $w(x, y)=\exp \left[i \boldsymbol{\alpha} c_{31} \psi_{3,1}(x, y)\right]$. 
Once more example of working of proposed model is considered if the incident wavefront is $w(x, y)=c_{31} \psi_{3,1}(x, y)$, $c_{31}=2 \sqrt{ } 2 \approx 2.8284$. The analyzer detects com-a aberration with coefficient $c_{31}^{\text {deteded }}=2.7489$. Resulting $2 \mathrm{D}$ amplitude patterns are shown in fig. 8 .

It should be noticed that aberrations detection can be successful only for weak aberrations of the wavefront. With increasing of aberrations linear approximation of the wavefront as a sum Zernike polynomials is becoming nonapplicable and false detection happens.

In the table 2 a dependence of detection quality is shown. Incident wavefront is constant and equal $w(x, y)=\exp \left[i \boldsymbol{\alpha} c_{31} \psi_{3,1}(x, y)\right], c_{31}=2 \sqrt{2} \approx 2.8284$. Parameter $\boldsymbol{\alpha}$ is varied from 0 to 2.0 ; we observe a varying of detected value $c_{31}^{\text {detected }}$ . From the table 2 it is seen that for too small and too high meanings of parameter $\alpha$ the detection is mistaken.

Table 2. Comparison of detected and initial aberration if initial wavefront has aberration $c_{31}=2.8284$ and parameter $\alpha$ varies from 0 to 2.0 .

\begin{tabular}{ccl}
\hline $\boldsymbol{\alpha}$ & $\boldsymbol{c}_{\mathbf{3 1}}$ & $\boldsymbol{c}_{31}^{\text {detected }}$ \\
\hline $\mathbf{0}$ & 2,8284 & 0,0104 \\
\hline $\mathbf{0 , 2 5}$ & 2,8284 & 1,6585 \\
\hline $\mathbf{0 , 5}$ & 2,8284 & 2,2136 \\
\hline $\mathbf{0 , 7 5}$ & 2,8284 & 2,5905 \\
\hline $\mathbf{1 , 0}$ & 2,8284 & 2,7489 \\
\hline $\mathbf{1 , 2 5}$ & 2,8284 & 2,6919 \\
\hline $\mathbf{1 , 5}$ & 2,8284 & 2,4603 \\
\hline $\mathbf{1 , 7 5}$ & 2,8284 & 2,1192 \\
\hline $\mathbf{2 , 0}$ & 2,8284 & 1,7398 \\
\hline
\end{tabular}

\section{Conclusions}

Every conventional aberration can be expressed in terms of decomposition into Zernike basis. Evidently, to compensate the most prominent aberration it is enough to modify the incident field by adding a phase which is complex-conjugated to revealed aberration. It can be done with diffractive optics methods including etching relief to the lenses surfaces or adding DOE to the optical system.

It is worth to notice that the size of proposed DOE from Zernike analyzer is equal about $5 \mathrm{~mm}$. 8-channel filter sensor has $512 \times 512$ pixels. Therefore it is easy to calculate resolution power of the device, it means $12.4 \mathrm{um}$.

However, resolution power of a diffractive optical element nowadays is limited by the 1 um meaning. Resolution of proposed 8-order DOE is far from technical limit. Thus, it says that the diffractive optical element can be easily produced with modern etching machines.

In this work provided simulation has shown that proposed Zernike analyzer can successfully detect aberration types and the model also reveals limitation of incident field aberration to be detected with the device. Defined tolerance range of aberration coefficient $\alpha$ is $\{0.5 ; 1.75\}$. Optimal meaning is 1 . If $\alpha$ goes out of tolerance range, aberration can not be detected or false detection can happens.

\section{Acknowledgements}

This work was supported by the Ministry of Education and Science of the Russian Federation and Russian Foundation for Basic Research grant No. 15-29-03823ofi_m.

\section{References}

[1] Goncharsky AV, Popov VV, Stepanov VV. Introduction to computer optics. Moscow: MSU Publishing House, 1991.

[2] Lane RG, Tallon M. Wave-front reconstruction using a Shack-Hartmann sensor. Appl. Opt. 1992; 31(32): $6902-6908$.

[3] Soifer VA, Golub MA. Laser beam mode selection by computer generated holograms. CRC Press, Boca Raton, 1994.

[4] Kotlyar VV, Khonina SN, Soifer VA. Light field decomposition in angular harmonics by means of diffractive optics. Journal of Modern Optics 1998; 45(7): $145-150$

[5] Khonina, SN, Almazov AA. Design of multi-channel phase spatial filter for selection of Gauss-Laguerre laser modes, Proceedings of SPIE 2002; 4705: 3039.

[6] Wolf E, Born M. Principles of Optics. Moscow: Science, 1973.

[7] Ha Y, Zhao D, Wang Y, Kotlyar VV, Khonina SN, Soifer VA. Diffractive optical element for Zernike decomposition. Proceedings of SPIE 1998; 3557: $191-197$.

[8] Khonina SN, Kotlyar VV, Soifer VA, Wang Y, Zhao D. Decomposition of a coherent light field using a phase Zernike filter. Proc. SPIE 1998; 3573: 550553.

[9] Khonina SN, Kotlyar VV, Wang Y. Diffractive optical element matched with Zernike basis. Pattern Recognition and Image Analysis 2001; 11(2): 442-445.

[10] Kotlyar VV, Khonina SN, Soifer VA, Wang Y, Zhao D. Coherent field phase retrieval us- ing a phase Zernike filter. Computer Optics 1997; 17 : 43-48.

[11] Khonina SN, Kotlyar VV, Kirsh DV. Zernike phase spatial filter for measuring the aberrations of the optical structures of the eye. Journal of Biomedical Photonics Engineering 2013; 1(2): 146-153.

[12] Zemax ${ }^{\circledR}$ User's Guide. Zemax Development Corporation, 2005.

[13] Khorin PA, Khonina SN, Karsakov AV, Branchevskiy SL. Analysis of corneal aberration of the human eye. Computer Optics 2016; 40(6): 810-817. DOI:

10.18287/0134-2452-2016-40-6-810-817.

[14] Kirilenko MS, Khorin PA, Porfirev AP. Wavefront analysis based on Zernike polynomials. CEUR Workshop Proceedings 2016; 1638: 66-75. DOI: 10.18287/1613-0073-2016-1638-66-75. 
Computer Optics and Nanophotonics / P.A. Khorin, S.A. Degtyarev

[15] Lombardo M, Lombardo G. Wave aberration of human eyes and new descriptors of image optical quality and visual performance. Journal of Cataract and Refractive Surgery 2010; 36: 313-331.

[16] Westheimer G, Liang J. Influence of ocular light scatter on the eye's optical performance. Journal of the Optical Society of America A. 1995; 12: 14171424.

[17] Tokovinin A, Heathcote S. DONUT: measuring optical aberrations from a single extrafocal image. Publications of the Astronomical Society of the Pacific 2006; 118(846): 1165-1175.

[18] Booth MJ. Wavefront sensorless adaptive optics for large aberrations. Optics Letters 2007; 32(1): 5-7.

[19] Klebanov IM, Karsakov AV, Khonina SN, Davydov AN, Polyakov KA. Wave front aberration compensation of space telescopes with telescope temperature field adjustment. Computer Optics 2017; 41(1): 30-36. DOI: 10.18287/0134-2452-2017-41-1-30-36.

[20] Porfirev AP, Khonina SN. Experimental investigation of multi-order diffractive optical elements matched with two types of Zernike functions. Proc. Optical Technologies for Telecommunications. SPIE 2016; 9807: 98070E-9 pp.

[21] Degtyarev SA, Porfirev AP, Khonina SN. Zernike basis-matched multi-order diffractive optical elements for wavefront weak aberrations analysis. Laser Physics and Photonics XVII; and Computational Biophysics and Analysis of Biomedical Data III. Proc. SPIE 2017; 10337: 103370Q. DOI:10.1117/12.2269218. 